\title{
The Prediction of Noise due to Jet Turbulence Convecting past Flight Vehicle Trailing Edges
}

\author{
Steven A. E. Miller* \\ The National Aeronautics and Space Administration
}

\begin{abstract}
High intensity acoustic radiation occurs when turbulence convects past airframe trailing edges. A mathematical model is developed to predict this acoustic radiation. The model is dependent on the local flow and turbulent statistics above the trailing edge of the flight vehicle airframe. These quantities are dependent on the jet and flight vehicle Mach numbers and jet temperature. A term in the model approximates the turbulent statistics of singlestream heated jet flows and is developed based upon measurement. The developed model is valid for a wide range of jet Mach numbers, jet temperature ratios, and flight vehicle Mach numbers. The model predicts traditional trailing edge noise if the jet is not interacting with the airframe. Predictions of mean-flow quantities and the cross-spectrum of static pressure near the airframe trailing edge are compared with measurement. Finally, predictions of acoustic intensity are compared with measurement and the model is shown to accurately capture the phenomenon.
\end{abstract}

\section{Nomenclature}

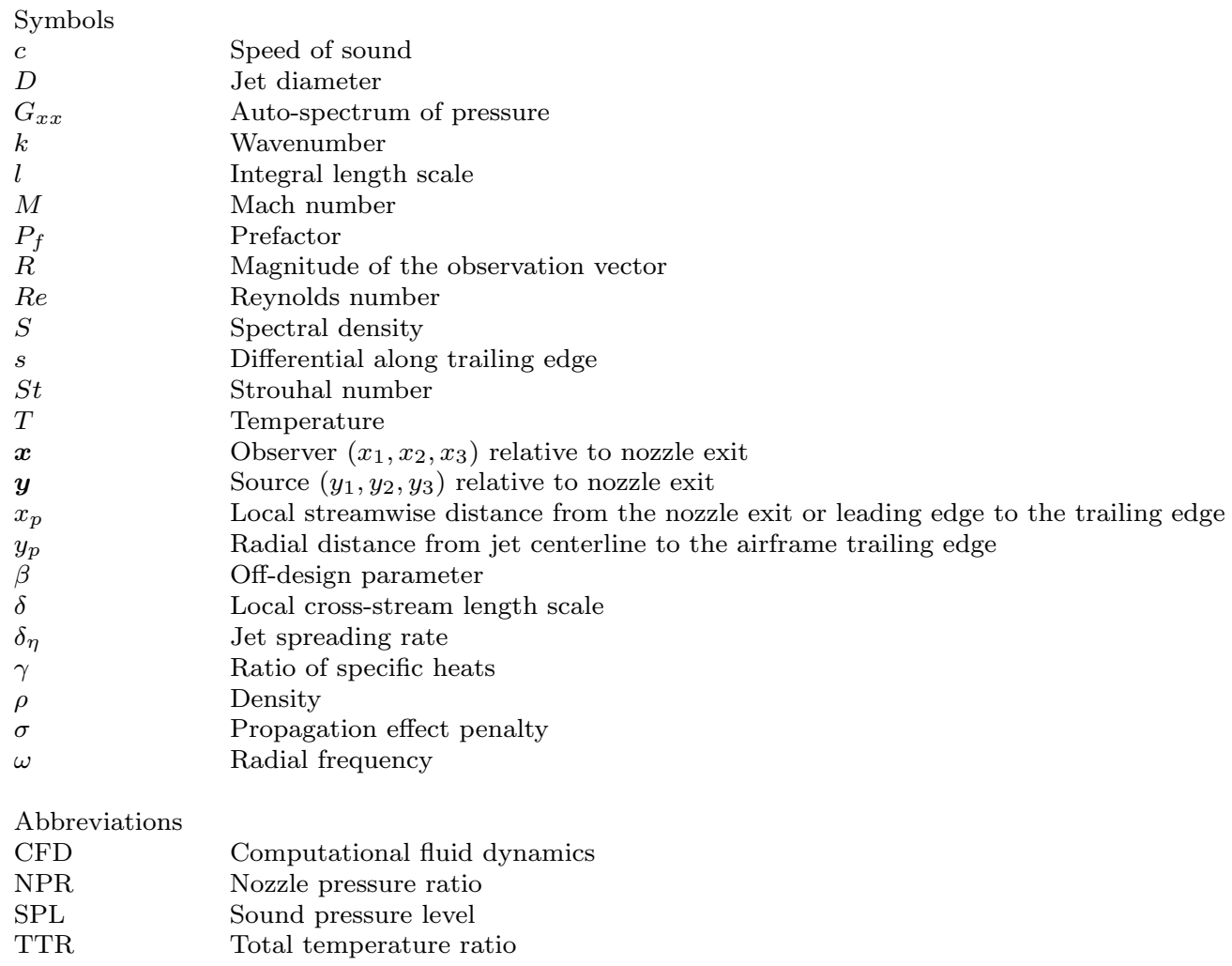

*Research Aerospace Engineer, Aeroacoustics Branch, NASA Langley Research Center, 2 N. Dryden St. MS 461, Hampton, VA, 23681, USA, AIAA Member, s.miller@nasa.gov 


\section{Introduction}

Jet engines are the predominant means of propulsion for flight vehicles. These vehicles include but are not limited to rockets, missiles, and military and civilian aircraft. First principle mathematical models and physical understanding of the jet and its associated noise has eluded investigators in the fields of fluid dynamics and aeroacoustics. The noise from jets is problematic and linked to flight vehicle failure, hearing loss, community annoyance, and psychological effects. When a jet engine is integrated with the airframe of a vehicle, the jet flow-field can be disturbed by the airframe surface. This disturbance induces a large amount of additional noise production relative to an isolated jet. Accurate prediction of the noise intensity and associated frequencies of the jet airframe interaction will yield physical insight into the unsteady aerodynamic source mechanism. It will also yield a valuable tool for aircraft designers that will enable them to minimize this noise source. In this paper, a model is created and validated for the prediction of the power spectral density resulting from the jet airframe trailing edge interaction. The model is valid for a wide range of jet Mach numbers, temperatures, and flight vehicle speeds.

This work draws heavily on previous experimental investigations and modeling efforts. Curle $^{1}$ examined the effects of noise from surfaces by extending Lighthill's ${ }^{2}$ theory. Later, Ffowcs Williams and Hawkings ${ }^{3}$ constructed generalized forms of the continuity and momentum equations involving two regions of the flowfield and created an acoustic analogy that involved the generalized density perturbation, a generalized Lighthill stress tensor, and two additional source terms that govern the sound from turbulence and the effect of moving surfaces. The latter two terms have characteristics associated with traditional acoustic monopole and dipole theory. Ffowcs Williams and Hall ${ }^{4}$ used Lighthill's theory to make predictions of trailing edge noise due to eddies near to and far from the airframe edge. Soon after the work of Ffowcs Williams et al.,${ }^{3,4}$ multiple models appeared in quick order. These included the predictive models of Jones, ${ }^{5}$ Chase, ${ }^{6}$ and notably Tam and $\mathrm{Yu}^{7}$ This later work by Tam and $\mathrm{Yu}^{7}$ was one of the first to experimentally and mathematically examine the noise from a rectangular jet flow convecting past a trailing edge. Spark schlieren images were produced of the jet trailing edge interaction. Amiet ${ }^{8,9}$ refined a model for the airframe trailing edge noise due to boundary layers. Trailing edge noise prediction models based on the pioneering work of Amiet were developed in experimental programs at NASA Langley by Brooks and Marcolini, ${ }^{10}$ Brooks et al. ${ }^{11}$ Brooks, ${ }^{12}$ Brooks et al.,${ }^{13}$ and Hutcheson and Brooks. ${ }^{14}$ Lawrence et al. ${ }^{15}$ modified the method of Amiet ${ }^{9}$ and included measured static pressure statistics along the trailing edge of the airframe. They showed that if the correct turbulent statistics were included within their model then accurate correlations

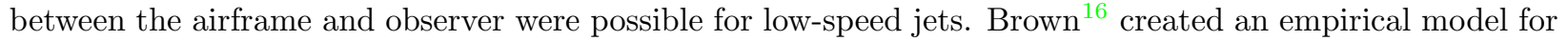
unheated subsonic jet structure interaction noise based upon master spectra and empirically fitted polynomial coefficients. Most recently, Goldstein et al. ${ }^{17}$ applied the well-known rapid distortion theory of turbulence to two-dimensional sheared mean-flows, and made preliminary predictions for a two-dimensional slotted jet flow convecting past a trailing edge. However, the theory does not capture the constructive interference patterns that are characteristic of this phenomenon and was only demonstrated for a single jet operating condition and airframe position. These theories, especially those of Tam and $\mathrm{Yu},{ }^{7}$ Amiet, ${ }^{8,9}$ Brooks and Marcolini, ${ }^{10}$ and Lawrence et $a l .{ }^{15}$ form the basis of this work.

The spectrum of jet noise is deceiving in its apparent simplicity but is extremely complicated due to the nature of the source. The source consists of compressible turbulence, shock-waves (for supersonic jets), and in the context of this paper, interactions with airframe surfaces. Figure 1 shows one such spectrum measured by Bridges and Brown ${ }^{18}$ and various predictions that correspond to the source mechanisms of jet noise. Sound pressure level (SPL) is on a per Strouhal number, St, basis where $S t$ is the non-dimensional frequency that is dependent on the fully expanded velocity and fully expanded jet diameter. The jet operates at a design Mach number, $M_{d}=1.50$, fully-expanded Mach number, $M_{j}=1.29$, and total temperature ratio, $\mathrm{TTR}=1.00$. The observer (microphone) is 100 jet diameters from the nozzle exit at an inlet angle, $\Psi=70^{\circ}$. The spectrum due to a free jet (static) is shown as the solid line with diamonds and its associated dominant aerodynamic sources are labeled. Mixing noise is present at all frequencies and occurs due to the turbulent mixing within the jet plume. For this particular off-design supersonic jet, it is dominant in the range of $0^{+}<S t \lesssim 0.4$ and typically peaks near $S t \approx 0.3$. Due to the semi-periodic shock-cell structure within the jet plume and the turbulence passing through it, broadband shock-associated noise (BBSAN) occurs and dominates the free jet spectrum in the range $S t \gtrsim 0.4$. Finally, the same shock-cell structure can support an aeroacoustic feedback loop within the plume that induces strong discrete (screech) tones that are observed at $S t \approx 0.4$ and 0.8 . The dashed-dot-dot line represents a prediction of the mixing noise by Miller, ${ }^{19}$ which is a modern acoustic analogy. The dashed-dot line represents a prediction of the BBSAN by Miller and Morris ${ }^{20}$ 
based on the model of Morris and Miller. ${ }^{21}$ The combination of these predictions result in an excellent model for the total jet noise over a wide range of jet Mach numbers and temperatures.

When the jet is placed near an airframe the power spectrum changes dramatically. In this example, the airframe (a large flat surface) extends 20 diameters downstream $\left(x_{p} / D\right)$ from the nozzle exit and extends 2 diameters $\left(y_{p} / D\right)$ from the nozzle centerline in the cross-stream direction. These effects can be observed in Fig. 1 by examining the differences between the measured jet and airframe spectrum (solid line with circles) of Brown ${ }^{22}$ and the free jet spectrum (solid line with diamonds) of Bridges and Brown. ${ }^{18}$ For $S t \gtrsim 0.2$ the total noise intensity is much lower because the mixing noise, BBSAN, and screech tone are 'shielded.' Specifically, the sound must propagate around the airframe. A prediction for jet-airframe mixing noise is shown as the dashed black line. This prediction is based on the preliminary work involving the tailored vector Green's function of the linearized Euler equations by Miller. ${ }^{23}$ A prediction for the jet-airframe BBSAN is not shown but can easily be conducted by the method of Miller and Morris ${ }^{20}$ combined with the tailored Green's function of Miller $^{23}$ for the airframe. At $S t \lesssim 0.2$ a large amount of additional acoustic intensity occurs that is due to the turbulence of the jet being deformed by the trailing edge of the airfoil. If the jet is farther away from the airframe, as discussed in the similarity analysis of Smith and Miller, ${ }^{24}$ then this jet-surface interaction noise will not be present. The solid black line is a prediction using the mathematical model and physical theory developed in this paper for jet-surface trailing edge noise.

In this paper, a model for the noise produced by the jet turbulence convecting past the airframe trailing edge is presented. The model is based on the work of Amiet ${ }^{8,9}$ and inspired by Tam and $\mathrm{Yu}^{7}$ and Lawrence $e t$ $a l .{ }^{15}$ The model depends on the integration of the source along the trailing edge of the flight vehicle airframe that includes the region where the jet turbulence is interacting with the trailing edge. The model seamlessly predicts both the noise due to jet turbulence near the airframe trailing edge and the trailing edge noise due to the ambient airflow (flight stream Mach number, $M_{\infty}$ ). The model scales correctly with the jet Mach number, jet temperature, flight stream Mach number, and is applicable to three-dimensional flows. It can easily be used for more complex flows from non-circular nozzles with the use of steady RANS solutions; however, the model presented here is independent of any external numerically generated results.

Development of a simple empirical mean-flow model is shown. Example predictions of the empirical mean-flow model and its relevance to the prediction model are discussed. Auto-spectrum of the fluctuating aerodynamic pressure above the airframe trailing edge is compared with measurement. Predictions are presented and compared to measurement for five different jet Mach numbers and a heated jet. The scaling of the acoustic intensity is compared with measurement as the jet centerline is moved farther from the airframe. Finally, the validity of the model and its limitations are discussed.

\section{Mathematical Model}

A model is developed for the spectral density of pressure due to turbulent jet impingement and trailing edge noise. The model is developed based on the work of Amiet ${ }^{8,9}$ and inspired by Lawrence et al. ${ }^{15} \mathrm{~A}$ key assumption is that the turbulence that convects past the airframe trailing edge, due to the jet flow and free-stream Mach number, is statistically stationary. Unlike previous theories, the noise from the jet impingement and trailing edge noise is due to the pressure fluctuations above the local turbulent boundary layer near the trailing edge.

Above the trailing edge of the airframe the fluctuating static pressure can be written in terms of its wavenumber, $\Delta p=\exp \left[i\left(\omega t-\omega y_{1} / u_{c}-k_{y_{2}} y_{2}\right)\right]$. See Amiet ${ }^{25}$ for the analytical development of $\Delta p$ from a sinusoidal turbulent velocity impulse. Following Amiet, ${ }^{9}$ for the pressure above the trailing edge of the airfoil, the method of Schwartzschild ${ }^{26}$ is used to calculate a Green's function. Two solutions are obtained, one for quadruples and the second for dipoles, and the latter is added to the former. A transfer function (Green's function) results that is used to obtain the far-field acoustic pressure by its convolution with a source. Due

to the stochastic nature of turbulence, we must relate the far-field acoustic pressure to the turbulent pressure statistics (cross-spectrum) rather than choose a deterministic approach. By using the same assumption as Amiet, ${ }^{9}$ that acoustic frequency is associated with a single streamwise turbulent wavenumber, we can form a solution that follows the same solution process as Amiet. ${ }^{8}$ This results in an analogous model that is similar to Amiet $^{9}$ (Eqn. 4a). The resultant spectral density, $S(\boldsymbol{x}, \omega)$ is, 


$$
S(\boldsymbol{x}, \omega)=\frac{P_{f}\left(x_{2}-y_{p}\right)^{2} \omega^{2}}{16 \pi^{2} c_{\infty}^{2}} \int_{-\infty}^{\infty} \frac{G_{x x} l_{\perp}|\mathcal{L}|^{2} x_{p}^{2}}{\sigma^{4}} d s
$$

where $c$ is the speed of sound, the constant $P_{f}=\left(10 \times 10^{4 / 5}\right)^{-1}$, the vector $\boldsymbol{x}=\boldsymbol{x}\left(x_{1}, x_{2}, x_{3}\right)$ is from the nozzle exit to the observer, $x_{p}$ is the streamwise distance from the nozzle exit along the centerline axis to a point above the trailing edge or the chord length, and $\omega$ is the radial frequency. The choice of $x_{p}$ will be apparent in the following analysis and is dependent on the trailing edge being 'wetted' by the jet or ambient flow. $y_{p}$ is the shortest radial distance from the nozzle centerline to the trailing edge. $d s$ is the differential of the line integral that is evaluated along the trailing edge of the airframe in the spanwise direction. Note that the terms in the integrand are dependent on $\boldsymbol{y}=\boldsymbol{y}\left(y_{1}, y_{2}, y_{3}\right)$, which is the vector from the nozzle exit to a point on $s$. Figure 2 illustrates some of these variables in the coordinate system. The remaining terms of Eqn. 1 are now explained.

Flight-stream propagation effects are partially taken into account by the denominator of the integrand,

$$
\sigma=\left[\left(x_{1}-y_{1}\right)^{2}+\beta^{2}\left(x_{2}-y_{2}\right)^{2}+\left(x_{3}-y_{3}\right)^{2}\right]^{1 / 2},
$$

where $\beta$ is the off-design parameter,

$$
\beta=\left(1-M_{\infty}^{2}\right)^{1 / 2},
$$

and $M_{\infty}$ is the free-stream (aircraft) Mach number based on the local ambient speed of sound, $c_{\infty} . l_{\perp}$ is the local integral scale of turbulence in the cross-stream direction $x_{2}$. This quantity varies along the trailing edge and corresponds to the cross-stream integral scale of turbulence just above the local boundary layer of the airfoil or within the jet plume above the trailing edge of the airfoil. We choose a model for $l_{\perp}$ as,

$$
l_{\perp}(\boldsymbol{y}, \omega)=\frac{\omega}{u_{c, p}}\left(\frac{\omega^{2}}{c_{\infty}^{2}}+\frac{\omega^{2}}{u_{c, p}^{2}}\right)^{-1},
$$

where $u_{c, p}$ is the local convection velocity of the coherent turbulent structures above the local boundary layer thickness or within the jet plume above the trailing edge. A subscript $p$ denotes the quantity just above the local boundary layer thickness, $\delta$, where $\delta$ is a function of $x_{2}$ and the local flow properties. This latter quantity is ideally found from experiment or numerical simulation. This model is similar to that of Amiet ${ }^{9}$ which varies based on the inverse of $\omega$ and the data of Willmarth and Roos. ${ }^{27}$ Here Eqn. 4 is based upon measured data of Brown and Wernet. ${ }^{28}$ We propose a model for $\delta$,

$$
\delta= \begin{cases}0.047 x_{p} R e_{l}^{-1 / 5} & \text { if } u(\boldsymbol{y}, \delta) \approx u_{\infty} \\ D_{j}\left(1+\alpha_{1} x_{p} / D_{j}\right) & \text { if } u(\boldsymbol{y}, \delta)>u_{\infty},\end{cases}
$$

where $\alpha_{1}=0.07$ is the spreading rate of the flow, $R e_{l}=\rho_{\infty} u_{\infty} c_{l} / \mu_{\infty}$ is the Reynolds number based on the local chord length, $c_{l}$, at $y_{2}, \rho$ is the density, and $\mu_{\infty}$ is the ambient kinematic viscosity. $D_{j}$ is the fully expanded jet diameter defined by Tam, ${ }^{29}$ that represents the equivalent jet diameter for fully-expanded jet flow,

$$
\frac{D_{j}}{D}=\left[\frac{1+\frac{\gamma-1}{2} M_{j}^{2}}{1+\frac{\gamma-1}{2} M_{d}^{2}}\right]^{\frac{\gamma+1}{4(\gamma-1)}}\left(\frac{M_{d}}{M_{j}}\right)^{1 / 2},
$$

where $D$ is the nozzle diameter and $\gamma$ is the ratio of specific heats. $D_{j}$ is equal to $D$ for subsonic jets. If a dual-stream or non-axisymmetric nozzle is present then the bypass or equivalent diameter can be used. The selection of $D_{j}$ is important because it is related linearly to the cross-stream length scale of the turbulence within the jet plume above the trailing edge of the airfoil as shown in Eqn. 5.

The auto-spectrum of the fluctuating static pressure at the surface is,

$$
\begin{array}{r}
G_{x x}(\boldsymbol{y}, \omega)=\delta \rho_{p}^{2} u_{p}^{3} \exp \left[-\left(\frac{(4 / 5) u_{j} / \delta+M_{j} u_{j} / \delta-\omega}{\sqrt{5} 2 \times 10^{4}}\right)^{2}\right] \\
\times\left(\frac{1}{8}+\frac{1}{8} \tanh \left[\frac{\omega}{20 \pi}-\frac{u_{p}}{500 D_{j f} M_{j}}\right]\right),
\end{array}
$$


which represents an empirical fit to measured data from the experiment of Brown and Wernet. ${ }^{28}$ The functional form is very different than that used by Amiet ${ }^{9}$ based upon data of Willmarth and Roos. ${ }^{27}$ Alternatively, data measured directly from experiment or found through numerical simulation can be used in place of $G_{x x}$.

The final factor of Eqn. 1, the integration of the response within the streamwise integral of the surface loading (analogous to $\mathrm{Amiet}^{8}$ ) is,

$$
\begin{aligned}
|\mathcal{L}|=\Theta^{-1} \mid(1+i)\{ & \left(\frac{M_{p}+2 x_{p} \omega\left(c_{p} \mu\right)^{-1}+1}{x_{1} / \sigma+1}\right)^{1 / 2} \exp [-2 i \Theta] E^{*}\left[\frac{2 \mu x_{1}}{\sigma}+2 \mu\right] \\
& \left.-E^{*}\left[2 \mu M_{p}+2 \mu+\frac{4 x_{p} \omega}{c_{p}}\right]\right\}-\exp [-2 i \Theta]+1 \mid \cos \left[\alpha_{2} \phi\right],
\end{aligned}
$$

which has been modified from the form originally proposed by Amiet. ${ }^{9} \mu$ is,

$$
\mu=\frac{1}{2} \frac{M_{p} x_{p} \omega}{u_{p} \beta^{2}},
$$

and $\Theta$ is,

$$
\Theta=2 \frac{x_{p} \omega}{c_{p}}+\mu\left(M_{p}-\frac{x_{1}}{\sigma}\right) .
$$

The function $E^{*}$ of Eqn. 8 is an integral of the form,

$$
E^{*}(\zeta)=\int_{0}^{\zeta}(2 \pi \xi)^{-1 / 2} \exp [-i \xi] d \xi .
$$

Equation 11 is in a well-known form that can easily be rewritten in terms of Fresnel integrals,

$$
\begin{aligned}
& E^{*}(\zeta)=\frac{1}{(2 \pi)^{1 / 2}} \int_{0}^{\zeta} \xi^{-1 / 2} \exp [-i \xi] d \xi \\
& =F_{c}\left[(2 / \pi)^{1 / 2} \zeta^{1 / 2}\right]-\frac{i \sqrt{\zeta^{2}}}{\zeta} F_{s}\left[(2 / \pi)^{1 / 2} \zeta^{1 / 2}\right],
\end{aligned}
$$

for arbitrary $\zeta$. The Fresnel integral pair is defined as,

$$
F_{c}(\Delta)=\int_{0}^{\Delta} \cos \left[\frac{\pi t^{2}}{2}\right] d t
$$

and,

$$
F_{s}(\Delta)=\int_{0}^{\Delta} \sin \left[\frac{\pi t^{2}}{2}\right] d t
$$

The Fresnel integrals can be written as a summation of two Error functions,

$$
F_{c}(\Delta)=\frac{1-i}{4}\left(\operatorname{erf}\left[\frac{1+i}{2} \pi^{1 / 2} \Delta\right]+\operatorname{erf}\left[\frac{1-i}{2} \pi^{1 / 2} \Delta\right]\right)
$$

and,

$$
F_{s}(\Delta)=\frac{1+i}{4}\left(\operatorname{erf}\left[\frac{1+i}{2} \pi^{1 / 2} \Delta\right]-\operatorname{erf}\left[\frac{1-i}{2} \pi^{1 / 2} \Delta\right]\right)
$$

where erf is the error function,

$$
\operatorname{erf}(x)=2 \pi^{-1 / 2} \int_{0}^{x} \exp \left[-t^{2}\right] d t .
$$

The argument of the error function appearing in the expansion of $F_{s}$ and $F_{c}$ is complex. The error function is available in tabular form and can easily be evaluated with many modern computing languages. It 
is often convenient (or necessary) to evaluate an error function with a complex argument using the Faddeeva function,

$$
w(z)=\exp \left[-z^{2}\right] \operatorname{erfc}[-i z]
$$

where $\operatorname{erfc}(z)$ is the complementary error function with complex argument $z$. Evaluation of the Faddeeva function can be performed numerically with the algorithm developed by Poppe and Wijers. ${ }^{30}$ erf is related to $w(z)$ by,

$$
\operatorname{erf}(z)=w[-i z] \exp \left[z^{2}\right]-1
$$

Equation 1 and its arguments are defined except for the mean-flow quantities. These quantities can easily be found with experimental or numerical investigations. Here, we will propose a simple empirical model for single-stream jets. The mean-flow model is valid for jet structure interactions in the range of $0^{+}<M_{j}<1.4$ and $1 \leq$ TTR $\lesssim 3.2$ and should be used with caution otherwise. It is in-part based on the models of Lau et $a l .{ }^{31}$ and Lau. ${ }^{32}$ The airframe structure has some effect on the jet mean-flow and development but for now we will assume that the plume development is axisymmetric. The core length $x_{c}$ of the jet is,

$$
x_{c}=D_{j}\left\{4.2+1.1\left(M_{j}-M_{\infty}\right)^{2}\right\},
$$

and the streamwise velocity component on the jet centerline axis is,

$$
u_{c}= \begin{cases}u_{j} & \text { if } y_{1}<x_{c} \\ \left(u_{j}-u_{\infty}\right)\left(1-\exp \left[\frac{1.35}{1-y_{1} / x_{c}}\right]\right)+u_{\infty} & \text { if } y_{1} \geq x_{c}\end{cases}
$$

and the static temperature on the jet centerline axis is,

$$
T_{c}= \begin{cases}T_{j} & \text { if } y_{1}<x_{c} \\ \left(T_{j}-T_{\infty}\right)\left(1-\exp \left[\frac{1.35}{1-y_{1} / x_{c}}\right]\right)+T_{\infty} & \text { if } y_{1} \geq x_{c} .\end{cases}
$$

The streamwise velocity component relative to the nozzle exit is,

$$
u\left(y_{1}, r\right)=\frac{\left(u_{j}-u_{\infty}\right)}{\left(u_{j}-u_{\infty}\right)} \frac{\left(1-\operatorname{erf}\left[\hat{\sigma} \eta^{*}\left(y_{1}, r\right)\right]\right)}{\left(1-\operatorname{erf}\left[\hat{\sigma} \eta^{*}\left(y_{1}, 0\right)\right]\right)}\left(u_{c}-u_{\infty}\right)+u_{\infty},
$$

where $r=\sqrt{y_{2}^{2}+y_{3}^{2}}$ and $\eta^{*}=\left(r-D_{j} / 2\right) / y_{1}$. The error functions have real arguments and the use of the Faddeeva function relation of Eqn. 18 is not required. The spreading rate of the jet is,

$$
\delta_{\eta}=0.177\left\{\left[1-0.294 M_{j}^{2}\right]\left[1+0.50\left(M_{j}^{2}-1\right)\left(T_{j} / T_{o}-1.40\right)^{2}\right]\right\},
$$

where $T_{o}$ is the stagnation temperature in the plenum of the nozzle. The temperature is required to find the Mach number. A similar equation for $T$ is created for this purpose,

$$
T\left(y_{1}, r\right)=\frac{\left(T_{j}-T_{\infty}\right)}{\left(T_{j}-T_{\infty}\right)} \frac{\left(1-\operatorname{erf}\left[\hat{\sigma}_{t} \eta^{*}\left(y_{1}, r\right)\right]\right)}{\left(1-\operatorname{erf}\left[\hat{\sigma}_{t} \eta^{*}\left(y_{1}, 0\right)\right]\right)}\left(T_{c}-T_{\infty}\right)+T_{\infty}
$$

where $\delta_{\eta t}=1.45 \delta_{\eta}$ and $\hat{\sigma}_{t}=\sqrt{\pi} / \delta_{\eta_{t}}$ is the Görtler spreading parameter. Quantities important for the developed model are the convection speed,

$$
\begin{aligned}
& u_{p}=u_{c}\left(x_{p}\right) \frac{u\left(x_{p},\left(y_{p}^{2}+y_{3}^{2}\right)^{1 / 2}\right)}{u\left(x_{p}, y_{p}\right)}\left(1+\frac{1}{2} \operatorname{erfc}\left[\frac{3}{2}-M_{j}^{3}\right]\right) \\
& \times \exp \left[-11\left(\left(y_{p}^{2}+y_{3}^{2}\right)^{1 / 2}-D_{j f} / 2\right)^{2}\right] \\
& \times \exp \left[-\ln [2]\left|\frac{u\left(x_{p},\left(y_{p}^{2}+y_{3}^{2}\right)^{1 / 2}\right)}{u\left(x_{p}, y_{p}\right)}\right|\right],
\end{aligned}
$$

the temperature $T_{p}=T\left(x_{p}, \sqrt{y_{p}^{2}+y_{3}^{2}}\right)$, local speed of sound $c_{p}=\sqrt{\gamma R T_{p}}$, and Mach number, $M_{p}=u_{p} / c_{p}$. The convection speed of the turbulence is assumed to be approximately $u_{c, p}=0.70 u_{p}$ with associated axial wavenumber $k_{x}=\omega / u_{c, p}$. The later assumption is required in the formation of Eqn. 1 . 


\section{Results}

This section discusses the coordinate system in relation to the previously defined independent variables within the mathematical development section. An example mean-flow prediction is shown based on the empirical model and compared with a steady RANS solution generated by a separate numerical method. The auto-spectrum model is compared with measurement. Then, example predictions are shown for a number of jet operating conditions and plate locations.

Figure 2 illustrates the problem geometry and coordinate system. The origin of the coordinate system is the nozzle exit. The $x_{1}$-axis coincides with the centerline axis of the nozzle, the $x_{2}$-axis is normal to and points away from the airframe, and the $x_{3}$-axis is in the cross-stream direction. $M_{\infty}$ is in the direction $x_{1}$. $y_{p}$ is the negative distance from the centerline axis to the trailing edge bounded on the $x_{2}$-axis and $x_{p}$ is the distance from the nozzle exit to the trailing edge bounded on the $x_{1}$-axis. The source vector, $\boldsymbol{y}$, is defined from the nozzle exit to a position along the airframe trailing edge defined by the line $s$. The differential element, $d s$, of the model equation is bounded on $s$ and $y$ is smallest at $\boldsymbol{y}=\boldsymbol{y}\left(x_{p},-y_{p}, 0\right)$. Note the trailing edge of the airframe is not restricted to a constant $y_{1}$ position and can vary in space. $\boldsymbol{x}$ is a vector from the nozzle exit to the observer. $\Psi$ is the angle between the downstream centerline nozzle axis and the vector $\boldsymbol{x}$, and $\phi$ represents the azimuthal angle about $x_{1}$. Observers with angle $\phi=0$ are restricted to the $x_{1}-x_{2}$ plane.

Tables 1 and 2 summarize the positions of the airframe and jet operating conditions, respectively, that are available to the author for validation. For each jet operating condition (row of Table 2), Table 1 shows various airframe positions relative to the nozzle exit. For every streamwise plate position, $x_{p} / D$, multiple cross-stream plate positions, $y_{p} / D$, are available. The first column of Table 2 states the nozzle name, Small Metal Chevron (SMC) and a three digit series number. Note neither nozzle has chevrons. Nozzle pressure ratio (NPR) is the ratio between the total pressure in the nozzle plenum and the ambient static pressure. The other quantities of Table 2 are defined in the previous section.

An example calculation of the variation of the mean-flow along the trailing edge, $s$, is shown in Fig. 3. The mean-flow quantities are induced by a $M_{j}=0.50$ and TTR $=1.00$ jet flow, $x_{p} / D=10$ and $y_{p} / D=-2$ airframe position, and $M_{\infty}=0$. This condition corresponds to the first row of Table 2 . The lines represent predictions using the developed empirical mean-flow model for single-stream jets. The symbols are from a respective steady RANS solution that includes the flat plate geometry and SMC000 nozzle relative to the plate. $u, T$, and $M$ are the streamwise velocity component, static temperature, and Mach number respectively above the local boundary layer thickness or above the local jet length scale in the $x_{2}$ direction. The solid line and diamonds represent $u$ and are predicted from the empirical model (Eqn. 23) and the steady RANS solution respectively. For this particular case the induced velocity due to the jet, according to the empirical model and the steady RANS solution, is less than $1 \mathrm{~m} / \mathrm{s}$. The largest magnitude of $u$ agrees very well with the steady RANS solution and both form a nearly Gaussian shape. The dash-dot line and circles represent $T$ and are predicted from the empirical model (Eqn. 25) and the steady RANS solution respectively. The ambient temperature is $293 \mathrm{~K}$. Because the jet is unheated, $T$ is less than the ambient temperature within the jet flow. The largest change in $T$ relative to the ambient is $0.1 \mathrm{~K}$ according to the model and $0.5 \mathrm{~K}$ according to the steady RANS solution. Like $u$, the shape is Gaussian but inverted about the ambient temperature. Heated or high speed cases induce very large variation in $T$ such as $M_{j}=1.50$ or $M_{j}=0.68(\mathrm{TTR}=1.93)$ in Table 2. The final mean-flow comparison shown in Fig. 3 is for $M$. The dashdot-dot line and triangles represent $M$ and are predicted from the empirical model and the steady RANS predictions respectively. $M$ is calculated by the ratio of Eqn. 23 and the speed of sound based upon Eqn. 25. In this case, the agreement between the model and steady RANS solution has the same characteristics as the agreement in the $u$ comparison.

Outside the range of $\left|x_{3} / D\right| \lesssim 2, u, T$, and $M$ are at nearly the ambient flight stream condition. Within the disturbed region of the mean-flow, the dominant noise is due to the jet structure interaction and outside this region traditional trailing edge noise dominates. These quantities and others continuously transition and the resultant sources of the jet-surface interaction and traditional trailing edge noise change with varying jet operating conditions, airframe position, and free-stream Mach number. Note that the quantities $u, T$, and $M$ can easily be provided by a steady RANS solver or another approach for potentially more accurate predictions. The induced quantities on the airframe trailing edge can be very small as illustrated in this case. Thus, the simple symmetric model is useful for many calculations and it will be shown that it works well for cases where the jet is highly deformed. One very important point must be stressed: the convection velocity and convective Mach number of the turbulent structures of the jet are much larger than the induced 
velocity and Mach number at the trailing edge.

The variation of the auto-spectrum of the fluctuating pressure above $\delta, G_{x x}$, as predicted by Eqn. 7 is compared with measurements in Fig. 4. The jet operates at $M_{j}=0.50$ and TTR $=1.00$ and the ambient Mach number is $M_{\infty}=0.001$. The airframe position is $y_{p} / D=-2$ and the streamwise trailing edge location $x_{p} / D$ is located at $4,8,10$, and 15 .

The measurements were conducted by Brown and Wernet $^{28}$ and the data were processed by Palumbo. ${ }^{33}$ Measurements at other conditions shown in Table 2 are not available to the author for validation of $G_{x x}$. The pressure sensor was located as close to the trailing edge as possible without compromising the structural integrity of the plate. For more information on the measurements see Brown and Wernet. ${ }^{28}$

Predictions using Eqn. 7 are shown as a solid, solid-dot, solid-dot-dot, and dashed lines for $x_{p} / D=$ 4, 8, 10, and 15 respectively. Processed measurements are shown as diamond, circle, triangle, and square symbols at the same respective locations as the predictions. There is a large amount of scatter present in the measured data. Measurements show that as $x_{p} / D$ increases, the magnitude and the spectral width increase. The frequency of the peak magnitude decreases with increasing $x_{p} / D$. Lower frequency spectral content increases in magnitude with increasing $x_{p} / D$ and is due to the growth of the turbulent scales with increasing streamwise distance. At large downstream locations, such as $x_{p} / D=15$, the overall width increases but intensity decreases.

These trends are generally captured by the proposed model, Eqn. 7. The model is linked to the jet operating condition and airframe position through a semi-empirical model of the statistical flow quantities. Predictions contain a low-frequency fall-off. The predicted high-frequency fall-off is too large relative to measurement, and a log-normal model can correctly capture this fall-off. The form of $G_{x x}$ is intended not to capture the log-normal fall-off of the measurement because the model must reflect the energy of noise radiated from a distance $\delta$ above the airframe trailing edge, and not the energy on the wall. The scaling terms present in $G_{x x}$ for Mach number and temperature effects are based purely on theory. It will be shown in the following noise predictions and comparisons that the choice of scaling terms is valid for the proposed model of $G_{x x}$.

Jet structure interaction noise predictions using Eqn. 1 are now compared with measurements of Brown. ${ }^{22}$ Predictions have been performed for all the airframe positions and jet conditions shown in Tables 1 and 2 respectively. The first prediction is shown in Fig. 5. Predictions of SPL per unit $S t$ are compared with measurements from the $M_{j}=0.50$ and TTR $=1.00$ jet. The nozzle is convergent $M_{d}=1.00$ (SMC000) and the plate location is $x_{p} / D=10$ and $y_{p} / D=-1$. Recall that the angle, $\Psi$, is measured from the upstream axis and is shown in each sub-figure. Free jet measurements of Bridges and Brown ${ }^{18}$ are shown as a line with diamond symbols and are labeled 'SHJAR' (Small Hot Jet Acoustic Rig). Jet with airframe measurements of Brown $^{22}$ are shown as a line with circles and are labeled 'JSIT' (Jet Structure Interaction Test). Predictions of the model equation are shown as a solid line without symbols. The free jet measurements (SHJAR) are shown along with the jet with airframe measurements (JSIT) to illustrate the spectral changes that occur when placing an airframe surface near the jet plume. Specifically, the free jet mixing and shock-associated noise can change dramatically by the presence of the airframe surface. Also, additional sources associated with the jet structure interaction noise are created by the introduction of the airframe into the flow. The predictions illustrate one type of jet structure interaction noise that is due to the turbulence being deformed by the plate trailing edge and its subsequent propagation via incident and diffracted waves. Predictions in this paper, particularly at low through mid $S t$, should be compared with the jet with airframe measurements (JSIT). The presented results are corrected for atmospheric absorption (lossless spectra) and corrected to a distance of 100 nozzle exit diameters $(R / D=100)$. Note that correcting for the atmospheric losses and propagation distance can be difficult for jets near airframes because the source is highly distributed and the distance that the acoustic waves travel to the observer can be difficult to calculate, especially so when the propagation mechanism primarily includes diffraction.

At the various observer angles the predicted spectral magnitude, spectral width, and fall-off at high and low frequencies is satisfactory predicted. However, in the upstream direction the spectral magnitude is lower than measurement by $4 \mathrm{~dB}$. The low-frequency fall-off matches measurement excellently in all cases, and this is observed even below $S t \approx 0.01$ when data are available. High frequency fall-off is also very satisfactory and there is little to no noise contribution above $S t \approx 0.8$. This is highly desirable because the spectrum above $S t \approx 0.8$ is dominated by the jet mixing noise, as shown and discussed in the previous section. In the sideline and upstream direction multiple peaks can be observed both in the prediction and measurement. These peaks are due to the diffraction of the noise by the trailing edge of the airframe and 
are taken into account by Eqn. 8. The first and dominant peak frequency, for this case, generally matches that of measurement. As the observer angle is increased the peak magnitude increases in frequency and the multiple broad spectral peaks are unified.

A second comparison is shown in Fig. 6 where $M_{j}=0.68$ and TTR $=1.93$. The nozzle is convergent $M_{d}=1.00$ (SMC000) and the plate location is $x_{p} / D=20$ and $y_{p} / D=-1$. Relative to the previously presented case the jet Mach number and temperature has increased and the plate position is extended. Multiple broad spectral humps are again observed both in the predictions and measurement and are more prevalent in the upstream and sideline observer directions. Jet mixing noise is more dominant at lower frequencies $(S t \approx 0.5)$ as the plate is extended. Predictions at angles $\Psi=110$ through 150 deg. match measurement. At $\Psi=50 \mathrm{deg}$. the prediction is approximately $8 \mathrm{~dB}$ lower than measurement in peak magnitude and lower in frequency by $S t \approx 0.03$. However, in this direction the multiple spectral peaks due to diffraction by the trailing edge are most apparent both in prediction and measurement. Although the peaks are not coincident in frequency with measurement, the respective predicted and measured peaks are present. Trends in prediction and measurement show that the narrower spectral width and increased magnitude in the upstream direction is due to a higher value of $x_{p} / D$ and not due to jet Mach number or increased temperature.

Figure 7 shows predictions and measurements of the $M_{j}=0.98$ and TTR $=1.00$ jet. The nozzle is convergent and the plate location is at $x_{p} / D=4$ and $y_{p} / D=-2$. Relative to the previous cases the nozzle is located very close to the plate edge. Also, comparing the free jet (SHJAR) and jet and airframe (JSIT) measured spectra relative to previous cases, shows that in the sideline through upstream direction there is very little change. At low- to mid-frequencies in the upstream and sideline directions there is only a very small increase in measured acoustic intensity due to the jet structure interaction. The predictions at these angles agree extremely well with measurement and correctly predict this small increase. The difference at higher frequencies is due to a type of shielding that is correctly predicted by Miller and Smith. ${ }^{34}$ At the downstream angles, $\Psi=130$ and 150 deg., the jet mixing noise dominates any contribution from the jet structure interaction noise. As expected, the predictions are completely dominated by the jet mixing noise. By comparing the predicted spectral shapes relative to previous cases shows that there are still multiple broad lobes present, however, the second broad loab is dominant in magnitude.

The next comparison is shown in Fig. 8 for an off-design $M_{j}=1.29$ and TTR $=1.00$ jet. This case is very similar to the one in the introduction as shown in Fig. 1 . The nozzle is convergent-divergent $M_{d}=1.50$ (SMC016) and the plate location is at $x_{p} / D=10$ and $y_{p} / D=-2$. Free jet spectra show all the characteristics of coherent and incoherent mixing noise and BBSAN. With the addition of the plate the measured spectra changes dramatically. These sources and changes can be predicted as discussed in the introductory section. Overall, mixing noise and BBSAN dominate all jet structure interaction noise above $S t \approx 0.4$. At the upstream and sideline direction, the peak jet structure interaction noise is predicted correctly in terms of magnitude and peak frequency. At lower frequencies and at $\Psi=50$ deg., the broad lobe is predicted correctly at $S t \approx 0.02$ but is $4 \mathrm{~dB}$ too low. The total noise at low frequencies in the downstream direction is a combination of the coherent jet mixing noise and jet structure interaction noise.

A final comparison of predictions relative to varying observer location is shown in Fig. 9 for the $M_{j}=1.50$ and TTR $=1.00$ jet. The nozzle is convergent-divergent $M_{d}=1.50$ (SMC016) and the plate location is at $x_{p} / D=20$ and $y_{p} / D=-2$. The jet operates supersonically and on-design. Unlike the previous case, the measured spectra only shows extremely weak BBSAN near $S t \approx 0.8$. At the sideline location, the predicted interference pattern is predicted but is 0 to $6 \mathrm{~dB}$ under-predicted (at the 'troughs'). Unfortunately, at upstream angles the under-prediction is worse. At $\Psi \geq 110 \mathrm{deg}$. the magnitudes and peak frequencies are predicted more accurately relative to the upstream angles. For example, at the extreme downstream angle the peak frequency and fall-off at high and low-frequencies is predicted correctly, relative to measurement. These previous two cases presented illustrate the on-set of compressible turbulence. This on-set has been mainly incorporated in this prediction method through empirical correction. Excellent measurements of the statistics of compressible turbulence and its onset for high speed jets near airframe surfaces can guide theorists to create superior predictions.

We will now examine the variation of the jet structure interaction noise by varying the radial position of the airframe. Figure 10 shows $M_{j}=0.68$ and TTR $=1.93$ jet spectra at $\Psi=90$ deg. and $R / D=100$ from the convergent SMC000 nozzle. The plate streamwise location is $x_{p} / D=10$ and $y_{p} / D$ is varied from -1 to -6. Each set of spectra consist of the free jet and JSIT measurement and associated prediction. Note that the free jet spectrum is the same for each plate position. The plate locations are labeled on the left and the 
maximum predicted SPL per unit $S t$ is shown above for each set.

As the plate is moved farther from the jet centerline axis the jet structure interaction noise intensity decreases. This can be observed by comparing the JSIT and SHJAR measurements in Fig. 10. Predictions at each $y_{p} / D$ location show the same relative trend as the SHJAR measurement. Also, the predictions at each $y_{p} / D$ location compare favorably relative to the JSIT measurement and illustrate decreasing amplitude and $S t$ with increasing jet structure separation.

The final comparisons of this paper are shown in Fig. 11 for the $M_{j}=1.29$ and TTR $=1.00$ jet. These comparisons are like the previous except the convergent-divergent $M_{d}=1.50$ (SMC016) nozzle is used and the jet is off-design and unheated. Examining the relative difference between the JSIT and SHJAR measurements shows similar trends to the heated subsonic case except for the strong presence of BBSAN. The low- and high-frequency fall-off and peak magnitudes show the same relatively good agreement with the JSIT measurements as previous cases. In this particular case, there are strong constructive peaks that are not observed in the measured spectrum, and this can possibly be attributed to data processing. The predictions relative to measurement in Figs. 10 and 11 strongly suggest that the model is capable of correctly predicting the fall-off of intensity and peak frequencies for subsonic, supersonic, and cold and hot jets. In summary, the model is validated for single-stream jet flows that operate from $M_{j}=0.50$ through $M_{j}=1.50$ and TTR $=1.00$ through TTR $=1.93$. There is no reason to expect that the intensity scaling will be incorrect for lower jet Mach numbers or higher temperatures.

\section{Conclusion}

A mathematical model is proposed for the prediction of noise from jet structure interaction. The model can seamlessly predict the jet structure interaction noise and noise induced by ambient air passing the trailing edge. A simple mean-flow model is developed and validated with steady RANS solutions, but steady RANS solutions based on more realistic nozzles can also be used for more accurate predictions. Furthermore, a model for the auto-spectrum of fluctuating pressure above the boundary layer is proposed and validated with measurement. The auto-spectrum model is dependent on the jet operating condition and local mean-flow. The jet structure interaction noise model is validated successfully for a wide range of available single-stream jet Mach numbers, temperature ratios, and airframe positions. Predictions compare very favorably with measurements at various observer angles. The decay of acoustic intensity with increasing jet centerline airframe distance also compares favorably.

The model is expected to scale correctly outside its range of validation with $M_{j}$ and TTR and can easily be extended to more complicated jet flows. However, careful measurements of compressible turbulent statistics within heated high-speed jet plumes are necessary to make successful predictions at higher jet Mach numbers and temperatures. These measurements will aid in the creation of a more general cross-spectrum model of fluctuating pressure. The author is hopeful that in the future such difficult measurements or even experiments will be undertaken. Finally, the combination of this source model with other models of the author yields an almost complete prediction method for off-design, heated, compressible turbulent jets.

\section{Acknowledgments}

This paper is inspired by the creative ideas of R. K. Amiet. Daniel L. Palumbo of the National Aeronautics and Space Administration (NASA) Langley Research Center is acknowledged for performing statistical analysis of fluctuating surface pressures of jet airframe interactions. James E. Bridges, Clifford F. Brown, and Mark P. Wernet of the NASA Glenn Research Center at Lewis Field are acknowledged for the use of their measurements. Alexandra Howton, a NASA Langley Research Center intern, is acknowledged for programing a preliminary model. The author is grateful for continuous support from the NASA Fundamental Aeronautics Program High Speed Project. 


\section{References}

${ }^{1}$ Curle, N., "The Influence of Solid Boundaries upon Aerodynamic Sound," Proc. Roy. Soc. A, Vol. 231, No. 1187, 1955, pp. 505-514. doi:10.1098/rspa.1955.0191.

${ }^{2}$ Lighthill, M. J., "On Sound Generated Aerodynamically. I. General Theory," Proc. R. Soc. Lond. A., Vol. 211, No. 1107, 1952, pp. 564-587. doi:10.1098/rspa.1952.0060.

${ }^{3}$ Ffowcs Williams, J. E. and Hawkings, D. L., "Sound Generation by Turbulence and Surfaces in Arbitrary Motion," Philosophical Transactions of the Royal Society of London. Series A, Mathematical and Physical Sciences, Vol. 264, No. 1151, May 1969, pp. 321-342. doi:10.1098/rsta.1969.0031.

${ }^{4}$ Ffowcs Williams, J. E. and Hall, L. H., "Aerodynamic Sound Generation by Turbulent Flow in the Vicinity of a Scattering Half Plane," Journal of Fluid Mechanics, Vol. 40, 1970, pp. 657-670. doi:10.1017/S0022112070000368.

${ }^{5}$ Jones, D. S., "Aerodynamic Sound due to a Source Near a Half Plane," Journal of the Institute of Mathematics and its Applications, Vol. 9, 1972, pp. 114-122. doi:10.1093/imamat/9.1.114.

${ }^{6}$ Chase, D. M., "Sound Radiated by Turbulent Flow off a Rigid Half-Plane as Obtained from a Wavenumber Spectrum of Hydrodynamic Pressure," Journal of the Acoustical Society of America, Vol. 52, 1972, pp. 1011-1022. doi:10.1121/1.1913170.

${ }^{7}$ Tam, C. K. W. and Yu, J. C., "Trailing Edge Noise," 2nd AIAA Aeroacoustics Conference, AIAA Paper 75-489, 1975. doi:10.2514/6.1975-489.

${ }^{8}$ Amiet, R. K., "Acoustic Radiation from an Airfoil in a Turbulent Stream," Journal of Sound and Vibration, Vol. 41, No. 4, 1975, pp. 407-420. doi:10.1016/S0022-460X(75)80105-2.

${ }^{9}$ Amiet, R. K., "Noise Due to Turbulent Flow Past a Trailing Edge," Journal of Sound and Vibration, Vol. 47, No. 3, 1976, pp. 387-393. doi:10.1016/0022-460X(76)90948-2.

${ }^{10}$ Brooks, T. F. and Marcolini, M. A., "Airfoil Self Noise - Effect of Scale," 8th AIAA Aeroacoustics Conference, April 11 - 13, Atlanta, Georgia, AIAA Paper 83-0785, 1983. doi:10.2514/6.1983-785.

${ }^{11}$ Brooks, T. F., Marcolini, M. A., and Pope, D. S., "Airfoil Trailing Edge Flow Measurements and Comparison with Theory Incorporating Open Wind Tunnel Corrections," 9th AIAA/NASA Aeroacoustics Conference, October 15 - 17, Williamsburg, Virginia, AIAA Paper 84-2266, 1984. doi:10.2514/3.9426.

${ }^{12}$ Brooks, T. F. and Marcolini, M. A., "Scaling of Airfoil Self-Noise Using Measured Flow Parameters," AIAA Journal, Vol. 23, No. 2, 1985, pp. 207-213. doi:10.2514/3.8896.

${ }^{13}$ Brooks, T. F., Marcolini, M. A., and Pope, D. S., "Airfoil Trailing-Edge Flow Measurements," AIAA Journal, Vol. 24, No. 8, 1986, pp. 1245-1251. doi:10.2514/3.9426.

${ }^{14}$ Hutcheson, F. V. and Brooks, T. F., "Measurement of Trailing Edge Noise Using Directional Array and Coherent Output Power Methods," 8th AIAA/CEAS Aeroacoustics Conference, June 17 - 19, Breckenridge, Colorado, AIAA Paper 2002-2472, 2002. doi:10.2514/6.2002-2472.

${ }^{15}$ Lawrence, J. L. T., Azarpeyvand, M., and Self, R. H., "Interaction Between a Flat Plate and a Circular Subsonic Jet," 17th AIAA/CEAS Aeroacoustics Conference, June 5 - 8, Portland, Oregon, AIA A Paper 2011-2745, 2011. doi:10.2514/6.2011-2745.

${ }^{16}$ Brown, C. A., "Developing an Empirical Model for Jet-Surface Interaction Noise," AIAA 52nd Aerospace Sciences Meeting, AIAA Paper 2014-0878, 2014. doi:10.2514/6.2014-0878.

${ }^{17}$ Goldstein, M. E., Afsar, M. Z., and Leib, S. J., "Non-Homogeneous Rapid Distortion Theory on Transversely Sheared Mean Flows," Journal of Fluid Mechanics, Vol. 736, No. 1187, 2013, pp. 532-569. doi:10.1017/jfm.2013.518.

${ }^{18}$ Bridges, J. and Brown, C. A., "Validation of the Small Hot Jet Acoustic Rig for Aeroacoustic Research," 11th AIAA/CEAS Aeroacoustics Conference, Monterey, California, 23 - 25 May, AIAA Paper 2005-2846, 2005. doi:10.2514/6.20052846.

${ }^{19}$ Miller, S. A. E., "Towards a Comprehensive Model of Jet Noise using an Acoustic Analogy and Steady RANS Solutions," 19th AIAA/CEAS Aeroacoustics Conference (34th AIAA Aeroacoustics Conference), Berlin, Germany, May 27-29, AIAA Paper 2013-2278, 2013. doi:10.2514/6.2013-2278.

${ }^{20}$ Miller, S. A. E. and Morris, P. J., "The Prediction of Broadband Shock-Associated Noise Including Propagation Effects," International Journal of Aeroacoustics, Vol. 11, No. 8, 2012, pp. 755-782. doi:10.1260/1475-472X.11.7-8.755.

${ }^{21}$ Morris, P. J. and Miller, S. A. E., "Prediction of Broadband Shock-Associated Noise Using Reynolds-Averaged NavierStokes Computational Fluid Dynamics," AIAA Journal, Vol. 48, No. 12, 2010, pp. 2931-2961. doi:10.2514/1.J050560.

${ }^{22}$ Brown, C. A., "Jet-Surface Interaction Test: Far-Field Noise Results," J. Eng. Gas Turbines Power, Vol. 135, No. 7, 2013, pp. 7-14. doi:10.1115/1.4023605.

${ }^{23}$ Miller, S. A. E., "The Prediction of Jet Noise Ground Effects using an Acoustic Analogy and a Tailored Green's Function," Journal of Sound and Vibration, Vol. 333, No. 4, 2014, pp. 1193-1207. doi:10.1016/j.jsv.2013.10.028.

${ }^{24}$ Smith, M. J. and Miller, S. A. E., "The Effects of Surfaces on the Aerodynamics and Acoustics of Jet Flows," $19 t h$ AIAA/CEAS Aeroacoustics Conference (34th AIAA Aeroacoustics Conference), Berlin, Germany, May 27-29, AIAA Paper 2013-2041, 2013. doi:10.2514/6.2013-2041.

${ }^{25}$ Amiet, R. K., "Compressibility Effects in Unsteady Thin-Airfoil Theory," AIAA Journal, Vol. 12, No. 2, 1974, pp. 253255. doi:10.2514/3.49212.

${ }^{26}$ Schwartzschild, K., "Die Beugung und Polarisation des Lichts Durch Einen Spalt," Mathematische Annalen, 1902, pp. $177-247$.

${ }^{27}$ Willmarth, W. W. and Roos, F. W., "Resolution and Structure of the Wall Pressure Field Beneath a Turbulent Boundary Layer," Journal of Fluid Mechanics, Vol. 22, 1965, pp. 81-94. doi:10.1017/S0022112065000599.

${ }^{28}$ Brown, C. A. and Wernet, M. P., "Jet-Surface Interaction Test: Flow Measurement Results," AIAA Aviation Conference, June 16 - 20, Atlanta, Georgia, AIAA Paper 2014, 2014.

${ }^{29}$ Tam, C. K. W., "Stochastic Model Theory of Broadband Shock-Associated Noise from Supersonic Jets," Journal of Sound and Vibration, Vol. 116, No. 2, 1987, pp. 265-302. doi:10.1016/S0022-460X(87)81303-2. 
${ }^{30}$ Poppe, G. P. M. and Wijers, C. M. J., "More Efficient Computation of the Complex Error Function," ACM Transactions on Mathematical Software (TOMS), Vol. 16, No. 1, 1990, pp. 38-46. doi:10.1145/77626.77629.

${ }^{31}$ Lau, J. C., Morris, P. J., and Fisher, M. J., "Measurements in Subsonic and Supersonic Free Jets using a Laser Velocimeter," Journal of Fluid Mechanics, Vol. 93, No. 1, 1979, pp. 1-27. doi:10.1017/S0022112079001750.

${ }^{32}$ Lau, J. C., Morris, P. J., and Fisher, M. J., "Effects of Exit Mach Number and Temperature on Mean-Flow and Turbulence Characteristics in Round Jets," Journal of Fluid Mechanics, Vol. 105, No. 1, 1981, pp. 193-218. doi:10.1017/S0022112081003170.

${ }^{33}$ Palumbo, D. L., Personal Correspondence, 2013.

${ }^{34}$ Miller, S. A. E. and Smith, M. J., "The Effects of Surfaces on the Aerodynamics and Acoustics of Jet Flows," 19th AIAA/CEAS Aeroacoustics Conference (34th AIAA Aeroacoustics Conference), Berlin, Germany, May 27-29, AIAA Paper 2013-2041, 2014. doi:10.2514/6.2013-2041.

\section{Tables}

Table 1. Plate Locations.

\begin{tabular}{|c|c|}
\hline \hline Axial Distance $\left(x_{p} / D\right)$ & Radial Distance $\left(y_{p} / D\right)$ \\
\hline 4 & $-1,-2,-4,-6,-8$, and -10 \\
\hline 10 & $-1,-2,-4,-6,-8$, and -10 \\
\hline 20 & $-1,-2,-4,-6,-8$, and -10 \\
\hline
\end{tabular}

Table 2. Jet Operating Conditions.

\begin{tabular}{|c|c|c|c|c|c|c|}
\hline \hline Nozzle & $M_{d}$ & NPR & TTR & $u_{j}(\mathrm{~m} / \mathrm{s})$ & $T_{j}(\mathrm{~K})$ & $M_{j}$ \\
\hline SMC000 & 1.00 & 1.186 & 1.00 & 167.4 & 279.0 & 0.50 \\
\hline SMC000 & 1.00 & 1.363 & 1.93 & 310.1 & 517.6 & 0.68 \\
\hline SMC000 & 1.00 & 1.850 & 1.00 & 312.9 & 244.3 & 0.98 \\
\hline SMC016 & 1.50 & 2.733 & 1.00 & 383.4 & 219.8 & 1.29 \\
\hline SMC016 & 1.50 & 3.671 & 1.00 & 427.4 & 202.1 & 1.50 \\
\hline
\end{tabular}

\section{Figures}

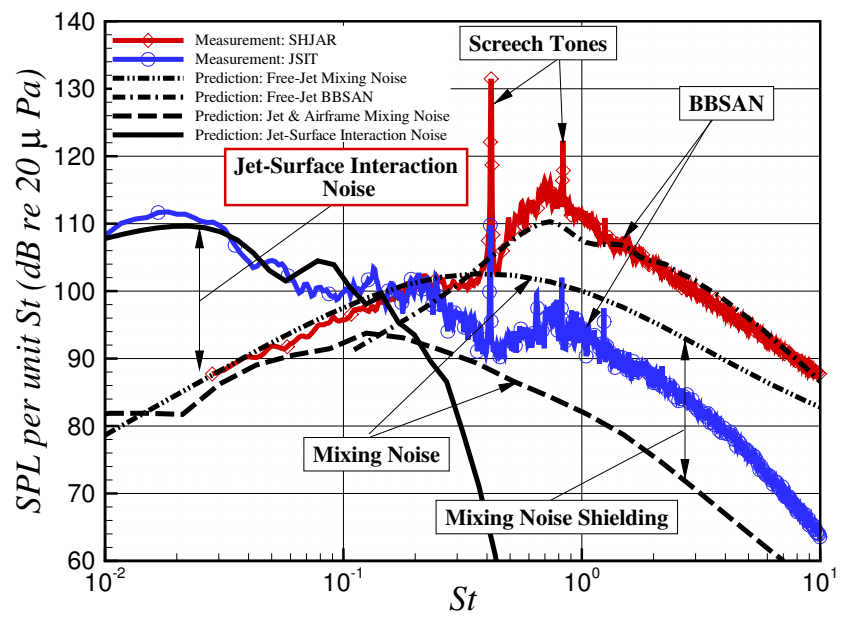

Figure 1. Predictions and measurements of the $M_{d}=1.50, M_{j}=1.29$, TTR $=\mathbf{1 . 0 0}$ jet at a distance of 100 jet diameters, $\psi=70 \mathrm{deg}$., on the opposite side of the airframe from the jet. The jet-airframe location is at $x_{p} / D=20$ and $y_{p} / D=-2$. 


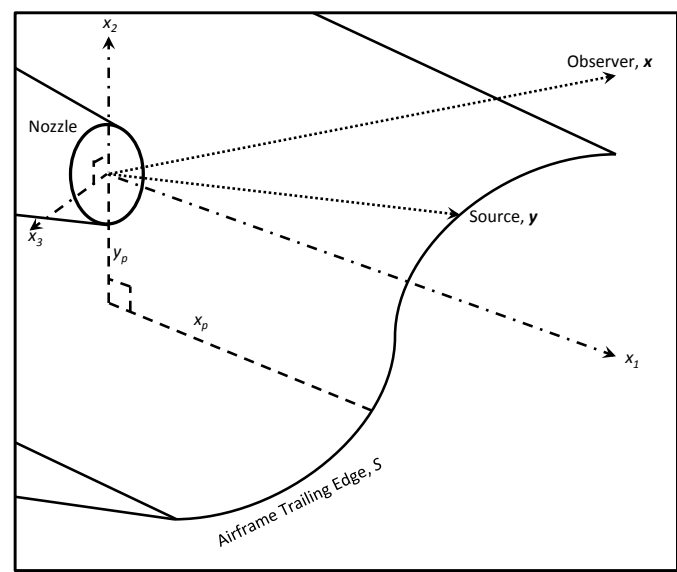

Figure 2. The coordinate system illustrating the nozzle off-set from the trailing edge of the airframe.

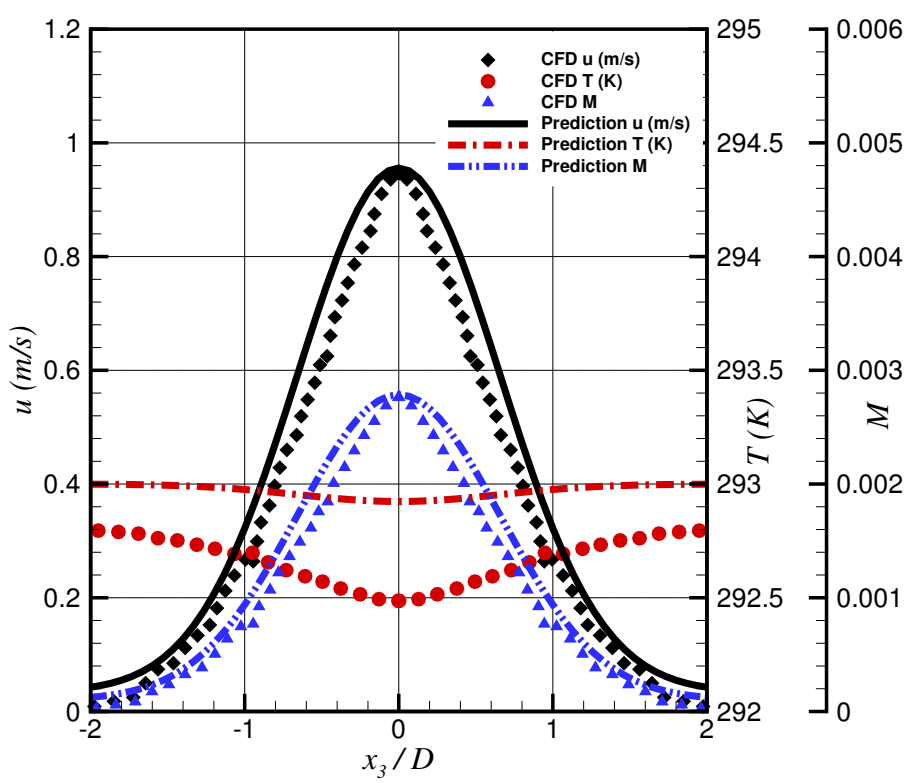

Figure 3. Predicted (lines) mean-flow quantities induced along the trailing edge from a $M_{j}=0.50$ and TTR $=1.00$ jet flow, $x_{p} / D=10$ and $y_{p} / D=-2$ airframe position, and $M_{\infty}=0$. The predictions are compared with steady RANS solutions (symbols) that include the flat plate geometry. 


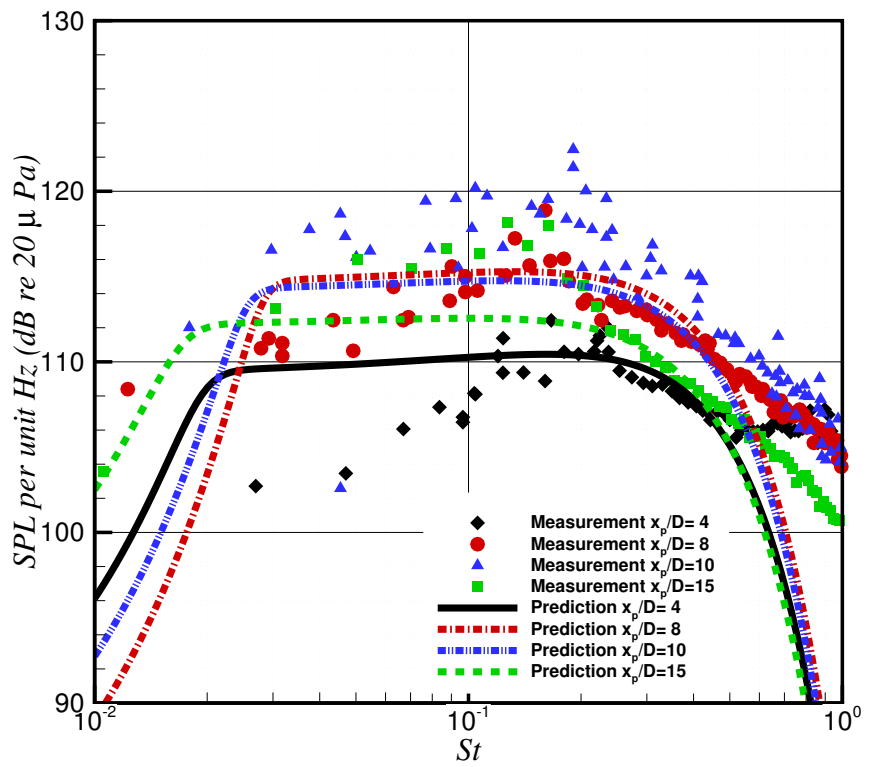

Figure 4. Predictions of $G_{x x}$ with measurement at the trailing edge induced by a $M_{j}=0.50$ and TTR $=1.00$ jet flow with varying $x_{p} / D$ and $y_{p} / D=-2$ airframe position, and $M_{\infty}=0.001$.
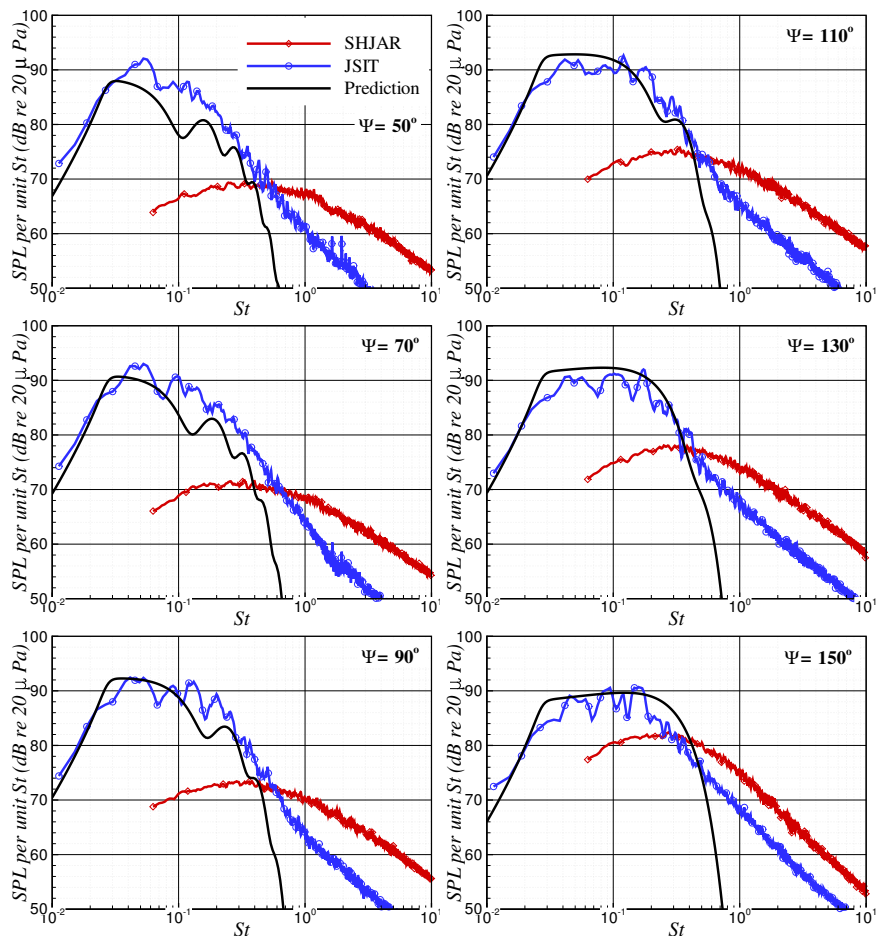

Figure 5. Predictions of SPL per unit $S t$ compared with measurements from the $M_{j}=0.50$ and TTR $=1.00$ jet. The nozzle is convergent $M_{d}=1.00$ (SMC000), the plate location is $x_{p} / D=10$ and $y_{p} / D=-1$, and microphones are at $R / D=100$ 

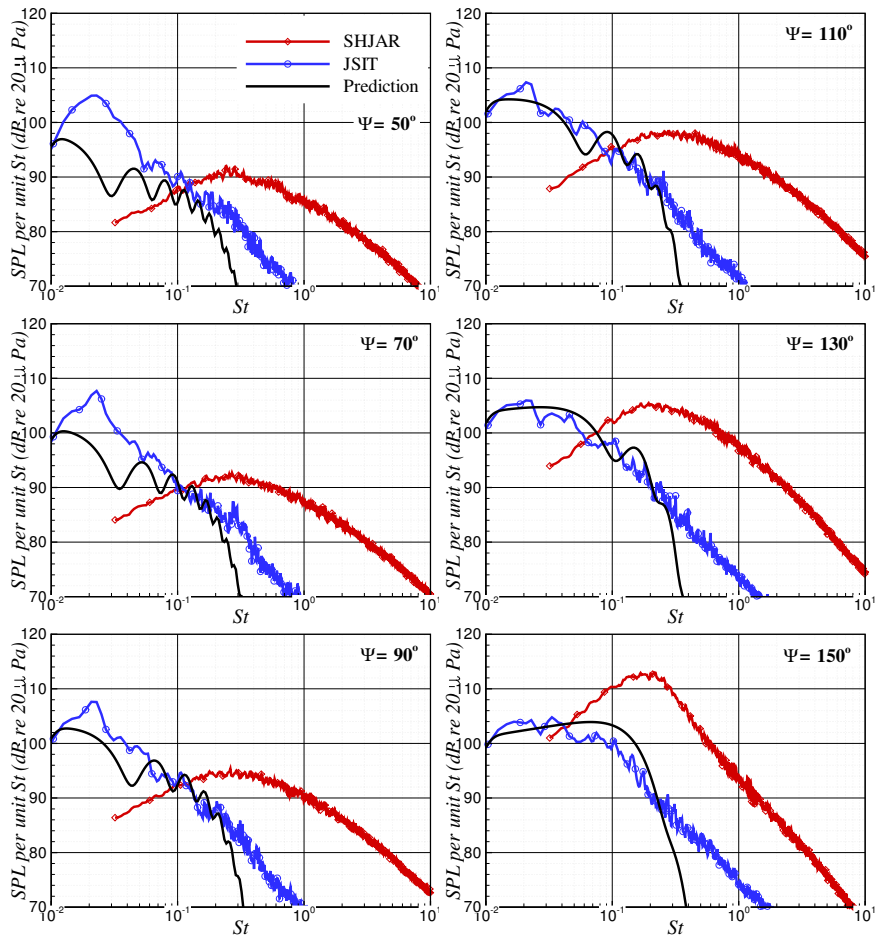

Figure 6. Predictions of SPL per unit $S t$ compared with measurements from the $M_{j}=0.68$ and TTR $=1.93$ jet. The

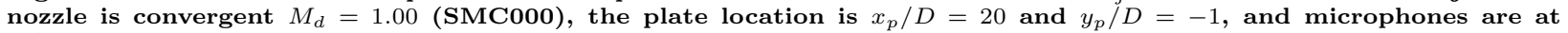
$R / D=100$
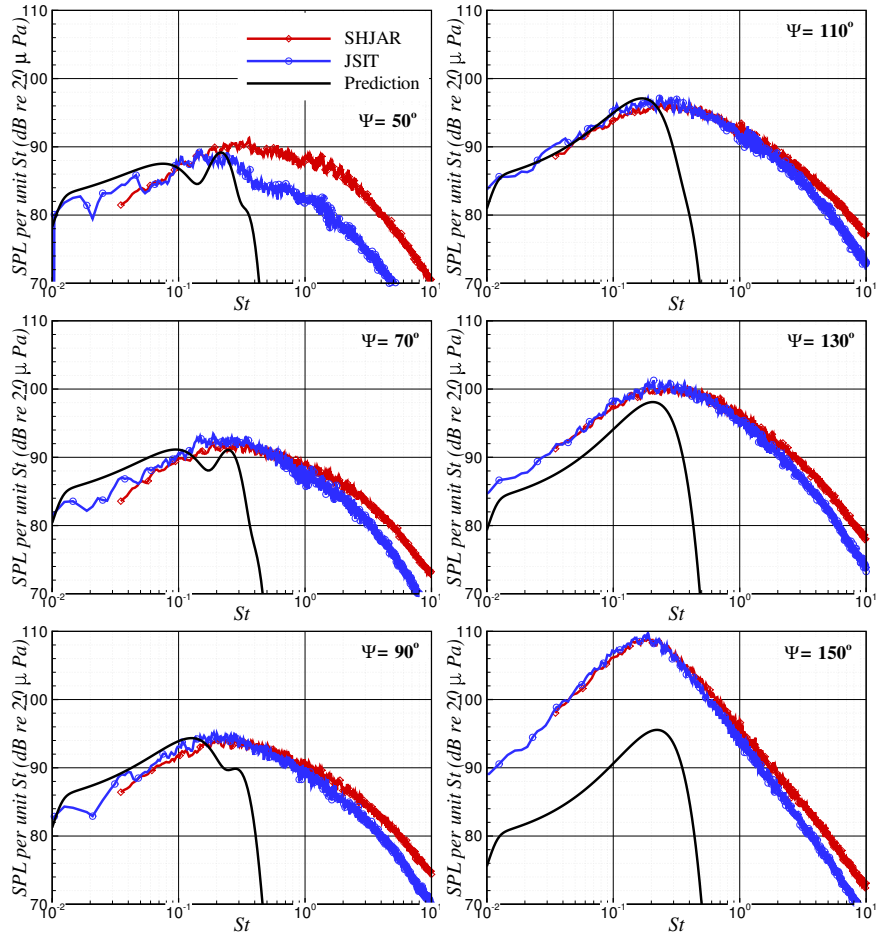

Figure 7. Predictions of SPL per unit $S t$ compared with measurements from the $M_{j}=0.98$ and TTR $=1.00$ jet. The nozzle is convergent $M_{d}=1.00$ ( $\mathrm{SMC000),} \mathrm{the} \mathrm{plate} \mathrm{location} \mathrm{is} x_{p} / D=4$ and $y_{p} / D=-2$, and microphones are at $R / D=100$. 

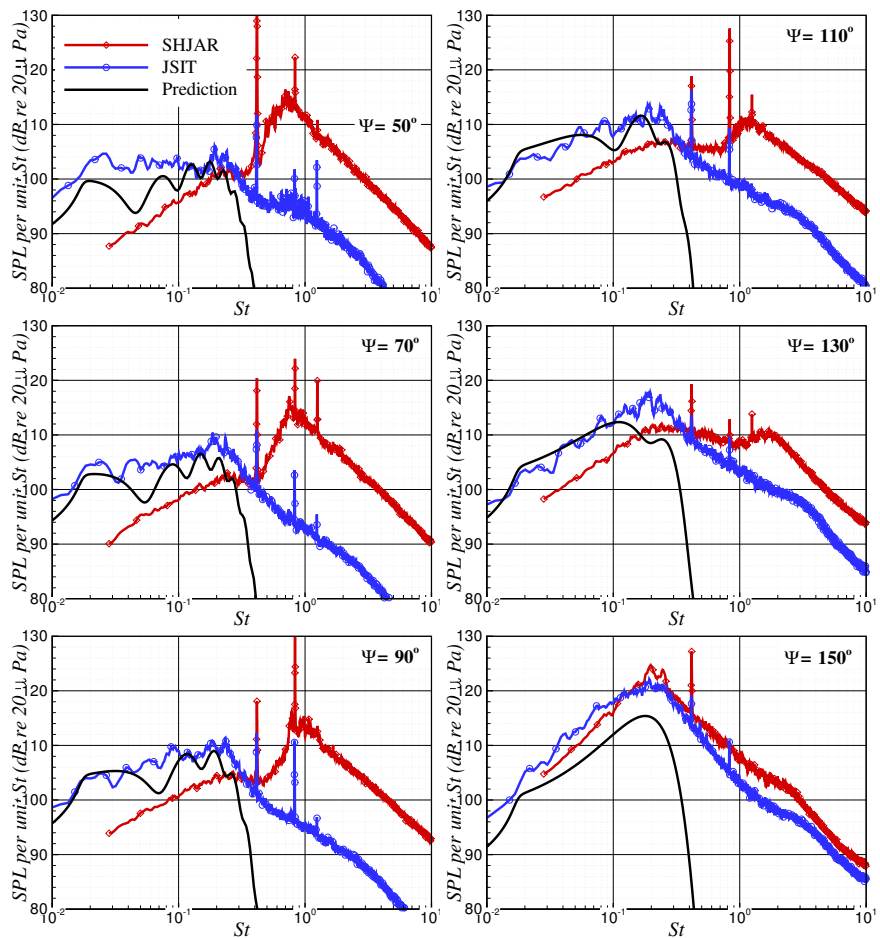

Figure 8. Predictions of SPL per unit $S t$ compared with measurements from the $M_{j}=1.29$ and TTR $=1.00$ jet. The nozzle is convergent-divergent $M_{d}=1.50$ (SMC016), the plate location is $x_{p} / D=10$ and $y_{p} / D=-2$, and microphones are at $R / D=100$.
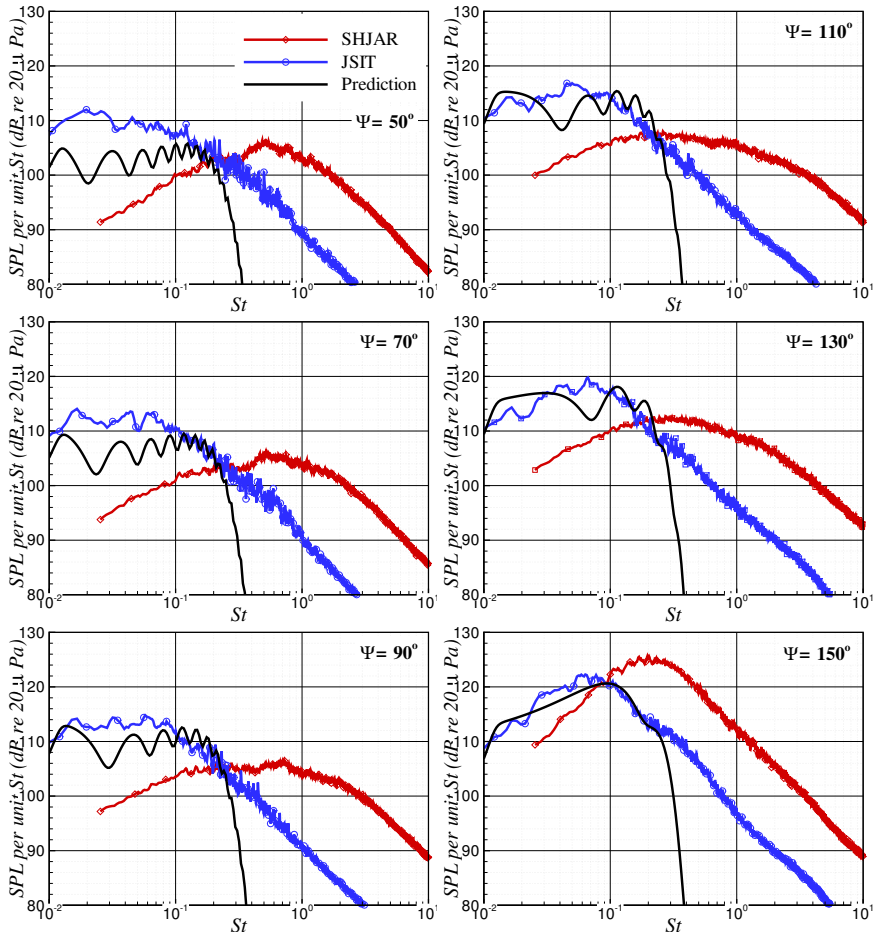

Figure 9. Predictions of SPL per unit $S t$ compared with measurements from the $M_{j}=1.50$ and TTR $=1.00$ jet. The nozzle is convergent-divergent $M_{d}=1.50$ (SMC016), the plate location is $x_{p} / D=20$ and $y_{p} / D=-2$, and microphones are at $R / D=100$. 


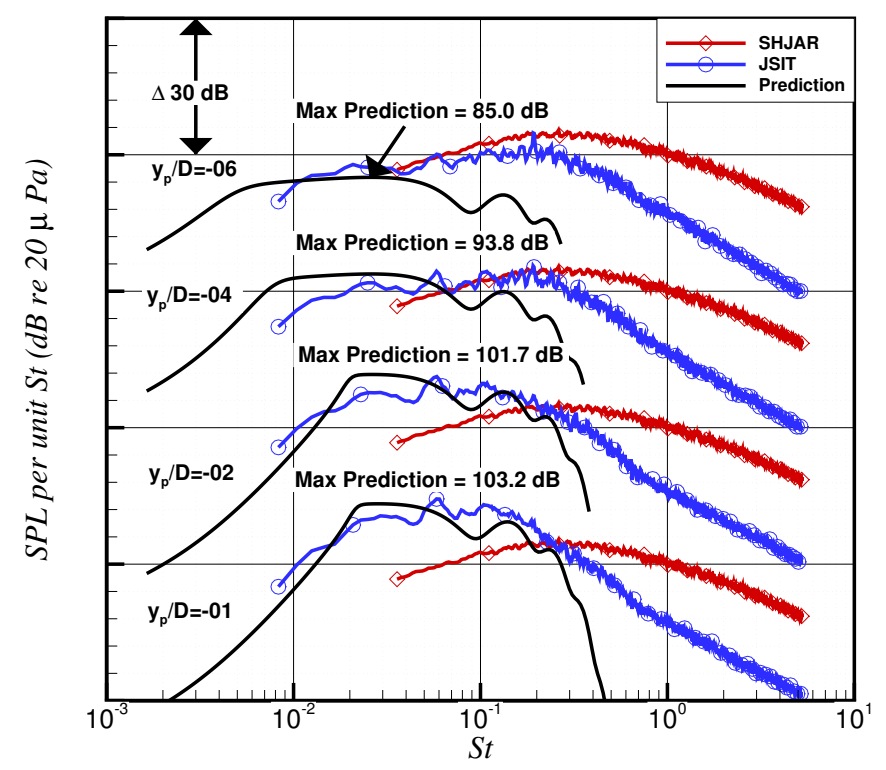

Figure 10. Predictions of SPL per unit $S t$ compared with measurements from the $M_{j}=0.68$ and TTR $=1.93$ jet. The nozzle is convergent $M_{d}=1.00$ (SMC000), the plate location is $x_{p} / D=10$ and $y_{p} / D$ is varied, and the microphone is at $R / D=100$ and $\Psi=90$ deg.

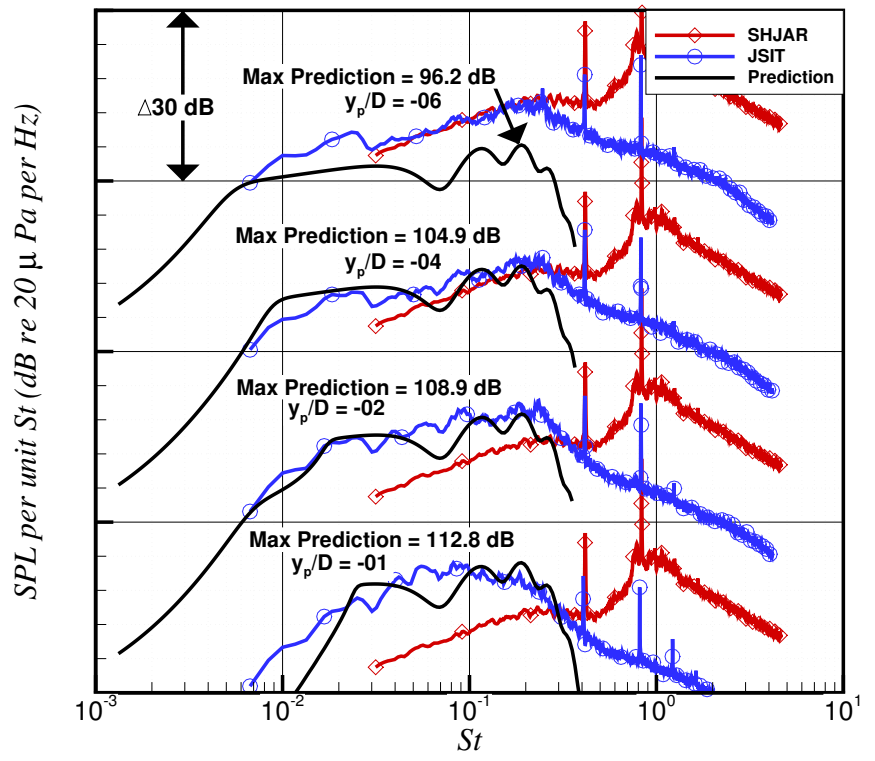

Figure 11. Predictions of SPL per unit $S t$ compared with measurements from the $M_{j}=1.29$ and TTR $=1.00$ jet. The nozzle is convergent-divergent $M_{d}=1.50$ (SMC016), the plate location is $x_{p} / D=10$ and $y_{p} / D$ is varied, and the microphone is at $R / D=100$ and $\Psi=90 \mathrm{deg}$. 